\title{
SATISFACCIÓN USUARIA RESPECTO A COMPETENCIA DE COMUNICACIÓN DEL PROFESIONAL DE ENFERMERÍA
}

\section{CUSTOMER SATISFACTION REGARDING COMMUNICATION QUALITY BY THE NURSING PROFESSIONAL}

\author{
Cecilia Landman Navarro* \\ María Jesús Cruz Osorio ** \\ Elizabeth García García ${ }^{* * *}$ \\ Paulina Pérez Meza ${ }^{* * * *}$ \\ PaUla SANDOVAl Barrera ***** \\ KarLa SeREy Burgos ${ }^{* * * * * *}$ \\ Camila Valdés Medina ${ }^{* * * * * *}$
}

\begin{abstract}
RESUMEN
El modelo de relación de persona a persona, de Joyce Travelbee, sustenta el estudio cuyo objetivo es determinar apreciación usuaria respecto a la competencia de comunicación del profesional de enfermería, considerando las dimensiones: proactividad, actitud profesional, comunicación verbal, comunicación no verbal y apoyo emocional. Estudio cuantitativo, descriptivo, transversal. Universo: 305 personas hospitalizadas, 3 servicios públicos, V Región, Chile, octubre y diciembre 2013. Unidades Mediana Complejidad. Muestra151, $\geq 18$ años; estadía $\geq 5$ días; Glasgow $=15$. Aceptación comité ética, consentimiento informado. Cuestionario satisfacción usuaria autoaplicado 17 ítemes escala Lickert 1-4. Alfa de Cronbach 0.943. Resultados: Alto grado de satisfacción global respecto de competencia de comunicación, las mujeres perciben una mayor insatisfacción que los hombres. Aumenta grado de satisfacción con mayor estadía; los jóvenes y quienes tienen mayor nivel de instrucción, mayor insatisfacción. La comunicación no verbal refleja menor nivel de satisfacción, en comparación con el resto de variables. La actitud profesional y proactividad se perciben en menor nivel que las otras dimensiones, siendo el apoyo emocional mejor valorado. Conclusiones: La comunicación y la visualización del otro como un ser único, autónomo, que tiene derecho a ser informado, tomar decisiones respecto a su salud, son dimensiones que deben ser respetadas. El centro del quehacer de la enfermera(o) como generador de cuidados a través de la comunicación, resaltando la relación persona a persona.
\end{abstract}

Palabras clave: Comunicación-barreras, comunicación-relaciones, enfermero-paciente-sistemas de comunicación en hospital.

\footnotetext{
*Enfermera-matrona, Directora de Posgrado y Postítulo Escuela Enfermería Universidad de Valparaíso, Chile. Email: cecilia. landman@uv.cl

${ }^{* *}$ Licenciada en Enfermería, Universidad de Valparaíso, Chile. Email: maje_77@hotmail.com

${ }^{* * *}$ Licenciada en Enfermería, Universidad de Valparaíso, Chile. Email: ely.garcia.garcia@gmail.com

${ }^{* * * *}$ Licenciada en Enfermería, Universidad de Valparaíso, Chile. Email: paulina.perez.meza@hotmail.com

${ }^{* * * * *}$ Licenciada en Enfermería, Universidad de Valparaíso, Chile. Email: polipops69@hotmail.com

${ }^{* * * * * *}$ Licenciada en Enfermería, Universidad de Valparaíso, Chile. Email: karla.sereyb@gmail.com

${ }^{* * * * * *}$ Licenciada en Enfermería, Universidad de Valparaíso, Chile. Email: camila.valdesm@hotmail.com
} 


\begin{abstract}
Joyce Travelbee's interpersonal relationship model sheds light on the study to measure customer rating regarding the quality of communication by the nursing professional, especially considering initiative, professional attitude, verbal communication, non-verbal communication and emotional support. Quantitative, descriptive, transversal study. Universe: 305 hospitalized persons at 3 public institutions, moderate complexity units. Sample size $151, \geq 18$ years old; stay $\geq 5$ days; Glasgow $=15$. Ethics Committee acceptance, informed consent, signed. Customer satisfaction self-questionnaire 17 items, Lickert scale 1-4. Cronbach alpha 0.943. Results: High degree of overall satisfaction regarding the quality of communication. However, female patients are relatively less satisfied than male patients. The degree of satisfaction increases with the hospitalization length. Young and well educated patients show the lowest relative satisfaction level. Non-verbal communication is less well developed than verbal communication. Professional attitude and initiative are the least developed aspects and emotional support the highest developed parameter. Conclusions: Communication and patient perception as a unique, autonomous being with the right to be well informed and make his/her own health decisions are all dimensions to be respected. These should be the focus of the nursing professional and also a source of patient care thus enhancing the interpersonal relationship.
\end{abstract}

Key words: Communication- communication barriers- nurse/patient relationship.

Fecha recepción: 10/10/13 Fecha aceptación: 30/12/14

\section{INTRODUCCIÓN}

El problema estudiado nace a partir de reflexiones y vivencias de los investigadores acerca de la importancia de la comunicación como una de las competencias del ser enfermero (1) así como también acerca de la valorización asignada a la comunicación enfermera(o) paciente por las personas que requieren de cuidados en el contexto hospitalario.

Desde ese punto se genera la siguiente pregunta investigativa ¿Cuál es el grado de satisfacción usuaria respecto a la competencia de comunicación del profesional de enfermería, en unidades de mediana complejidad médico-quirúrgica?

El contexto en el cual se desarrolló el estudio considera tres hospitales públicos, en unidades de mediana complejidad médico-quirúrgica, las cuales son responsables de atenciones médicas básicas y de especialidad y de cuidados de enfermería básicos y medianamente complejos, sin requerir de utiliza- ción de alta tecnología para la realización de procedimientos, como es la terapia endovenosa, medición de parámetros vitales; control de balance hídrico, administración de medicamentos por diversas vías; curaciones, cuidados físicos en caso de limitaciones del paciente, entre otros. El sistema de turnos de las enfermeras es de "cuarto turno" el primer día en horario de 8:00 a 20:00 h; el segundo día 20:00-8:00; el tercero y cuarto días libres, para continuar con la secuencia. Existe una enfermera en turnos fijos de 8:00 a 17:30 los días hábiles, para coordinaciones y apoyo de la unidad. Por cada enfermera existen entre 4 ó 5 técnicos paramédicos que apoyan al profesional en el cuidado de pacientes.

Este estudio tiene como propósito relevar el papel de la comunicación enfermera(o)-paciente, como un indicador de calidad de la atención en el proceso de cuidar. $\mathrm{La}$ calidad medida a través del grado de satisfacción usuaria, o grado de concordancia entre las expectativas de calidad y la atención recibida por parte del proveedor de cuidados de salud; algunos estudios referentes a la calidad 
de los servicios de salud consideran que en este proceso intervienen tres aspectos, como es el técnico-instrumental, el comunicativo y las comodidades de la asistencia (14, 16-21, 40, 44).

La comunicación interpersonal es considerada como un acto de humanidad que congrega al profesional de enfermería y a quienes éste atiende en un ambiente de confianza y afectividad mutua $(2,3)$, por lo que se requiere indagar acerca de cómo ésta se da en la práctica, estableciendo como constructo teórico, para su medición, las siguientes dimensiones: proactividad, actitud profesional, comunicación verbal, comunicación no verbal y apoyo emocional $(4,5)$. La comunicación entendida como una herramienta para disminuir el sufrimiento, favorecer la comprensión, la aceptación y la operatividad del tratamiento.

El profesional de enfermería, cuando interactúa con el otro, se constituye en un importante instrumento terapéutico, relevando su manera de atender y animar a los pacientes para su recuperación $(6,7)$ y como señalaba Florence Nightingale en sus notas sobre enfermería: “...una enfermera debe ser una persona de la que se pueda uno fiar, en otras palabras, capaz de ser enfermera de confian$\mathrm{za} "(8)$

Travelbee $(2,9,10)$ señala que en el proceso de cuidar, la enfermera utiliza todos sus recursos personales y profesionales para establecer lo que ella llama una "relación persona a persona", donde ambas son consideradas como seres únicos, insertos en un contexto social y cultural que brinda una particularidad a esta relación intersubjetiva y a sus significados, de ahí que la comunicación se establece a partir de una escucha activa por parte del profesional de enfermería, que permita establecer los nexos necesarios para comprender al otro en su real dimensión, requiere además de una conexión emocional y una aceptación del otro.

Es importante señalar que en la actualidad, cada vez más se reporta una creciente despersonalización en las relaciones humanas, esto también se ve reflejado en las relaciones enfermera-paciente. Por otra parte, la existencia de una población cada vez más informada que identifica y defiende sus derechos, asignándole a la comunicación con el equipo de salud y en especial con el profesional de enfermería, un valor fundamental como indicador de calidad de la atención, (11- 15).

Las revisiones realizadas acerca de la percepción usuaria de la calidad señalan, de manera fundamental, aspectos tales como el grado de preparación del equipo de salud, no específicamente a la(al) enfermera(o), cómo es su presentación personal, la calidad de las instalaciones, el acceso a los servicios y prestaciones de salud, el periodo de demora en la atención, la seguridad (22-27); sin embargo, un menor número de estos se relacionan con tópicos atinentes a la comunicación enfermera-paciente, como es el trato digno, la amabilidad, la confianza, la credibilidad, el interés, la empatía, la escucha activa, la utilización de un lenguaje comprensivo y la privacidad $(28,30,37)$.

En Chile, actualmente los servicios de salud se han visto inmersos en un modelo que promueve la gestión de la calidad institucional, producto de lineamientos ministeriales reflejados en la reforma de salud y de un sistema de acreditación certificado por la superintendencia de salud, sistema que se focaliza hacia la protección de los derechos y satisfacción del usuario $(12,13,27)$.

Por esto, hoy en día es esencial que los profesionales de la salud desarrollen competencias que permitan entregar a las personas el mayor grado de calidad de atención, cuidados humanizados y seguros, con los recursos disponibles para ello; contando además con habilidades comunicativas, técnicas y administrativas, para contribuir en el proceso de recuperación de las personas (28-39, 41-43, 46, 47). Correspondiéndole al profesional de enfermería fomentar una relación interpersonal con los pacientes, demostran- 
do comprensión, respeto y reciprocidad, incorporando elementos de la comunicación y escucha activa, adaptando el lenguaje a su propia idiosincrasia, sin levantar barreras idiomáticas, que permita a las personas percibir a un profesional involucrado genuinamente en su atención $(35,43,46,47)$, generando, por tanto, uno de los cambios más radicales en la calidad de la atención en el contexto hospitalario.

\section{MATERIALES Y MÉTODO}

El presente estudio se realizó por medio de un abordaje cuantitativo, descriptivo, transversal, cuyo objetivo fue determinar el grado de satisfacción usuaria respecto a la competencia de comunicación del profesional de en- fermería, en el contexto hospitalario. Instrumento "Cuestionario para la identificación del grado de satisfacción usuaria percibida respecto a la competencia de comunicación del profesional de enfermería" diseñado por los autores, basados en otros cuestionarios de medición de satisfacción como Care Q (49) y GATHA (50) y en los principios orientadores de la teoría de Joyce Travelbee. Cuestionario autoadministrado para la medición de la comunicación interpersonal enfermera-paciente, consta de 17 ítemes con graduación en escala apreciación Lickert 1-4 de cada una de los ítemes $(1=$ muy insatisfecho y $4=$ muy satisfecho), construido con las variables: proactividad, actitud profesional, comunicación verbal, comunicación no verbal y apoyo emocional (Tabla 1). Variables sociodemográficas: sexo, edad, escolaridad, días de hospitalización, unidad o servicio.

Tabla 1. Dimensiones de la comunicación (variables medidas)

\begin{tabular}{|c|c|c|}
\hline Dimensiones & Indicador & Ítemes \\
\hline \multirow{5}{*}{ PROACTIVIDAD } & Identificación & $\begin{array}{l}\text { 1. ¿Las enfermeras(os) se presentan a Ud. antes de realizarle al- } \\
\text { gún procedimiento? }\end{array}$ \\
\hline & Tiempo de respuesta & $\begin{array}{l}\text { 2. ¿Cuándo usted solicitó la atención de las enfermeras(os), acu- } \\
\text { dieron a su llamado? }\end{array}$ \\
\hline & Educación & $\begin{array}{l}\text { 3. ¿Las enfermeras }(o s) \text { responden a sus preguntas cuando tiene } \\
\text { dudas? }\end{array}$ \\
\hline & & $\begin{array}{l}\text { 4. ¿Las enfermeras(os) le dan indicaciones sobre su propio cui- } \\
\text { dado? }\end{array}$ \\
\hline & & $\begin{array}{l}\text { 5. ¿Las enfermeras(os) le entregan información necesaria para } \\
\text { que tome decisiones en relación a su salud? }\end{array}$ \\
\hline \multirow{5}{*}{$\begin{array}{l}\text { ACTITUD } \\
\text { PROFESIONAL }\end{array}$} & Empatía & $\begin{array}{l}\text { 1. ¿Las enfermeras(os) se muestran comprometidas(os) con sus } \\
\text { cuidados? }\end{array}$ \\
\hline & Interés & $\begin{array}{l}\text { 2. ¿Las enfermeras(os) comprenden lo que le pasa o se pone en } \\
\text { su lugar? }\end{array}$ \\
\hline & Respeto & 3. ¿Las enfermeras(os) respetan sus decisiones y opiniones? \\
\hline & Disposición & $\begin{array}{l}\text { 4. ¿Las enfermeras(os) tienen la disposición necesaria al mo- } \\
\text { mento de atender sus necesidades? }\end{array}$ \\
\hline & Capacidad de Resonancia & \\
\hline \multirow{2}{*}{$\begin{array}{l}\text { COMUNICACIÓN } \\
\text { VERBAL }\end{array}$} & Preguntas directas & $\begin{array}{l}\text { 1. ¿Considera que las preguntas que le realizan las enferme- } \\
\text { ras(os) son claras y precisas? }\end{array}$ \\
\hline & Información oportuna y clara & $\begin{array}{l}\text { 2. ¿Son claras las indicaciones o instrucciones que le han entre- } \\
\text { gado las enfermeras }(\mathrm{os}) \text { ? }\end{array}$ \\
\hline
\end{tabular}


Continuación Tabla 1 .

\begin{tabular}{|c|c|c|}
\hline \multirow{5}{*}{$\begin{array}{l}\text { COMUNICACIÓN } \\
\text { NO VERBAL }\end{array}$} & Tono de voz & $\begin{array}{l}\text { 1. ¿Las enfermeras(os) son capaces de transmitirle tranquilidad } \\
\text { y confianza mediante su expresión facial (gestos) y tono de voz? }\end{array}$ \\
\hline & Lenguaje corporal & $\begin{array}{l}\text { 2. ¿Las enfermeras(os) son capaces de calmarlo mediante el con- } \\
\text { tacto físico (darle la mano, tocar el hombro)? }\end{array}$ \\
\hline & Contacto físico & 3. ¿Las enfermeras(os) lo miran a los ojos mientras le hablan? \\
\hline & Contacto visual & $\begin{array}{l}\text { 4. ¿Las enfermeras(os) lo escuchan atentamente cuando usted } \\
\text { les habla? }\end{array}$ \\
\hline & Escucha activa & \\
\hline \multirow{2}{*}{$\begin{array}{l}\text { APOYO } \\
\text { EMOCIONAL }\end{array}$} & Compañía & $\begin{array}{l}\text { 1. ¿Las enfermeras(os) lo acompañan durante los procedimien- } \\
\text { tos que se le realizan? }\end{array}$ \\
\hline & Confianza & $\begin{array}{l}\text { 2. ¿Las enfermeras(os) le inspiran confianza en cuanto a la aten- } \\
\text { ción que le brindan las Enfermeras(os)? }\end{array}$ \\
\hline
\end{tabular}

$\mathrm{Al}$ instrumento se le agregaron 2 preguntas abiertas que permitieron discriminar si las personas encuestadas identificaban al profesional de enfermería (17).

La confiabilidad del instrumento medida con el estadígrafo Alfa de Cronbach tuvo un valor global de 0,94 , presentando un excelente índice de consistencia interna, permitiendo comprobar que el instrumento es fiable con mediciones estables y consistentes.

El universo corresponde a 305 personas hospitalizadas, durante los meses de octubre a diciembre 2013, en tres servicios públicos de la Región de Valparaíso, Chile, en unidades de mediana complejidad médico-quirúrgica. La muestra fue intencionada, de acuerdo a la temporalidad del estudio; quedando constituida por 151 personas, quienes consintieron participar y que cumplieron con los criterios de inclusión (edad $\geq 18$ años; estadía: $\geq 5$ días; Glasgow $=15$ puntos). Como otro criterio de exclusión se consideró a pacientes que no identificaban al profesional de enfermería.

Los datos recabados se incorporaron a una matriz Microsoft Excel 2007, para el análisis se utilizó programa SPSS versión 15. Estadígrafos utilizados, distribución de frecuencias, media de datos agrupados, promedios, porcentajes. Los criterios para la realización del análisis de la información: El rango que refleja el grado de satisfacción global de la competencia de comunicación es entre 17 y 68 puntos. A cada una de las variables se les otorgó un puntaje de acuerdo al grado de satisfacción, 1.0 a 1.7 (muy insatisfecho), 1.8 a 2.7 (insatisfecho), 2.8 a 3.3 (satisfecho) y 3.4 a 4.0 (muy satisfecho).

Aspectos éticos: Consentimiento informado y firmado, aprobación del comité de ética hospitalario. Autorización autores de instrumentos de base para su adaptación.

\section{RESULTADOS}

La caracterización de la muestra: existe una distribución similar entre los sexos, el rango de edad que lidera es el de los adultos mayores, en segundo lugar los adultos; en cuanto a la escolaridad, lidera la educación media completa y básica incompleta, en menor proporción educación superior completa y superior incompleta. Existiendo una distribución similar entre los servicios de medicina y cirugía.

Los días de mayor permanencia son entre 5 y 15 días y en menor grado 31 días o más.

Los resultados respecto al grado de satisfacción global de la comunicación del profesional de enfermería percibida por los pacientes muestran índices positivos en las tres instituciones estudiadas, los valores de satis- 
facción oscilan entre 73 y 100\%, considerando los valores de satisfecho y muy satisfecho.

$\mathrm{Al}$ analizar los datos de acuerdo a las variables de caracterización, las mujeres muestran mayores niveles de insatisfacción en relación a los hombres, siendo solo ellas quienes manifestaron un mayor grado de insatisfacción (Gráfico 1). Los adultos jóvenes y adultos manifiestan mayor nivel de insatisfacción que los jóvenes y adultos mayores.

Gráfico 1. Nivel de satisfacción global en relación al sexo.

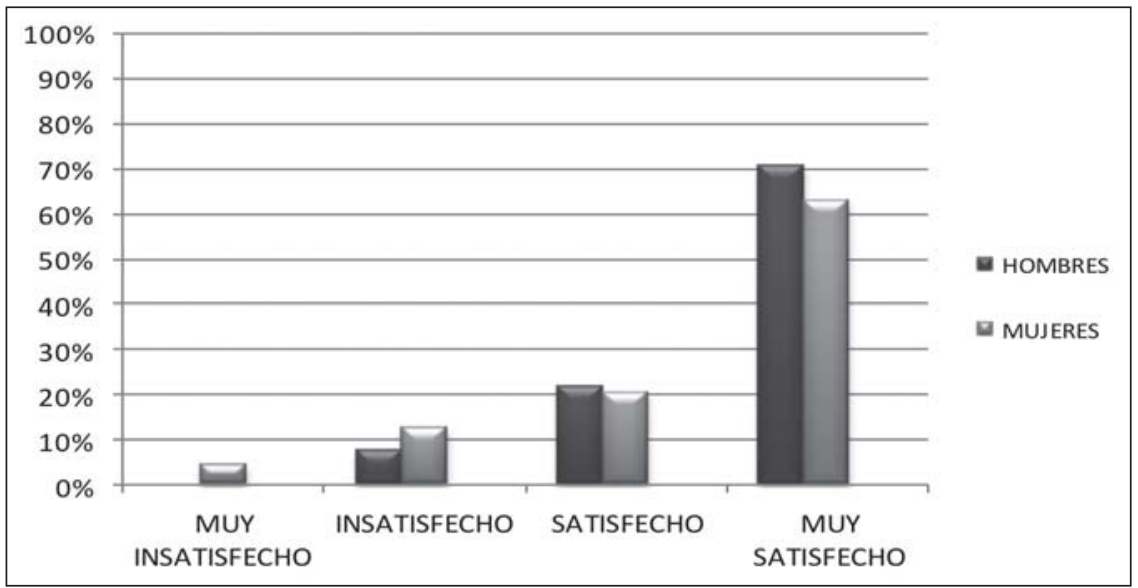

En relación a la escolaridad, quienes tienen enseñanza media completa y superior incompleta presentan un mayor grado de insatisfacción que las personas con niveles inferiores de escolaridad.

En los centros de salud estudiados, sin importar el servicio, se da la tendencia que a menor tiempo de estadía, menor grado de satisfacción de las personas encuestadas. Se observa que quienes están en servicios de cirugía, cuya estadía es más corta, muestran un nivel de insatisfacción más alto que quienes están en servicios de medicina.

Respecto del nivel de satisfacción de la proactividad del profesional, la cual ha sido medida en sus dimensiones de identificación del profesional, tiempo de respuesta a solicitudes de información y entrega de educación, los resultados indican que existe un alto nivel de satisfacción en dos de los tres hospitales estudiados, uno de ellos (H2) presentó niveles de insatisfacción moderados, siendo una de las áreas menormente ponderadas la educación entregada por el profesional (Gráfico 2).

El nivel de satisfacción general en relación a la actitud profesional, medida de acuerdo a la empatía, respeto e interés por el otro, presenta una valoración alta, considerando percepciones de muy satisfecho y satisfecho.

Nivel de satisfacción general en relación a la comunicación verbal, en dos de los tres centros estudiados (H1 y H3) se observa un alto grado de satisfacción, sin embargo $\mathrm{H} 2$ con valores más altos de insatisfacción.

Nivel de satisfacción general en relación a la comunicación no verbal, los usuarios la valoran por sobre otros aspectos, presentando un alto nivel de insatisfacción (Gráfico 3).

Nivel de satisfacción general en relación al apoyo emocional, una de las áreas mejor percibidas por las personas encuestadas, es la referida a la confianza y compañía. 
Gráfico 2. Nivel de satisfacción global según proactividad.

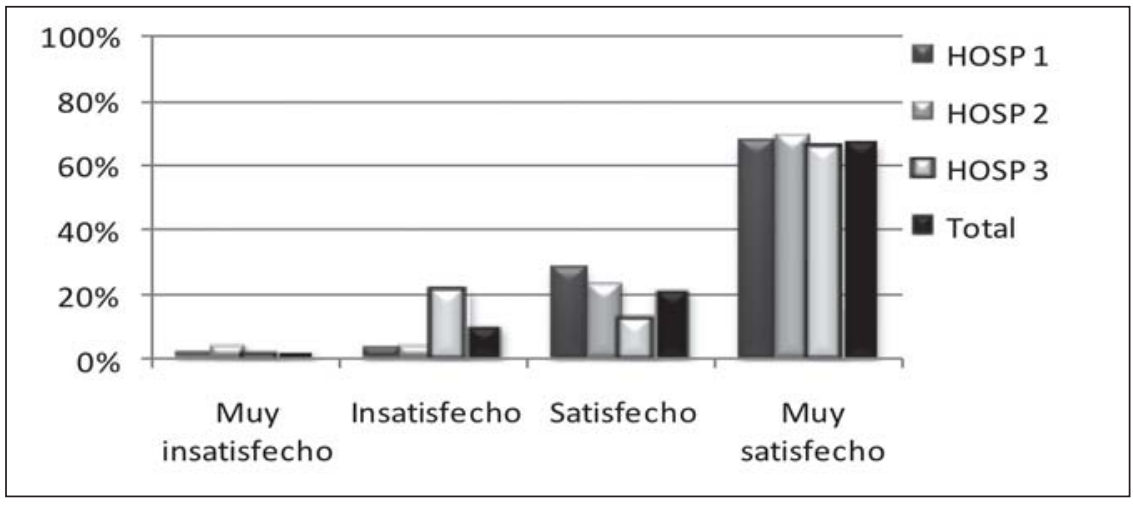

Grafico 3. Nivel de satisfacción general.

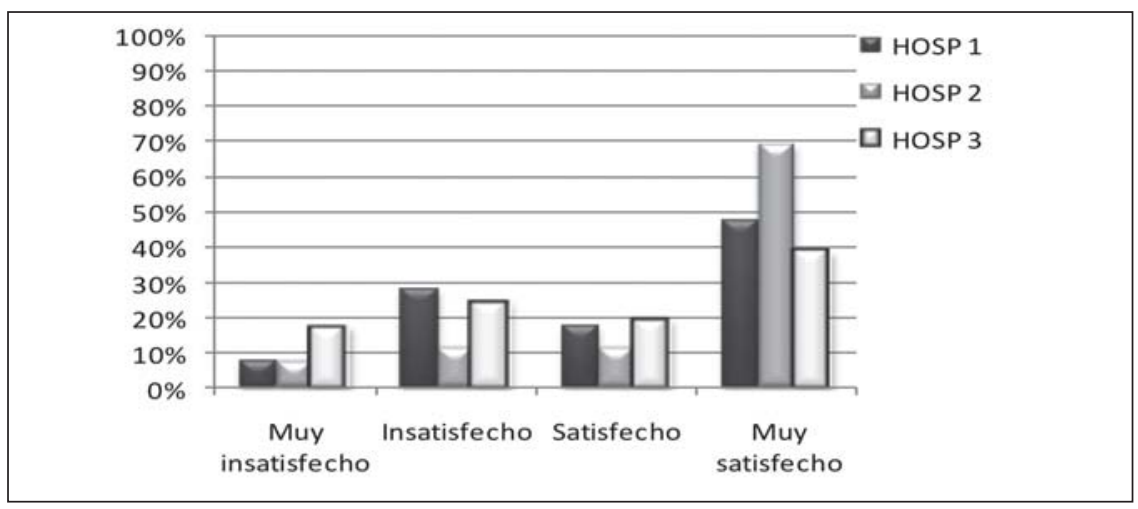

\section{DISCUSIÓN Y CONCLUSIÓN}

El presente estudio realizado en un contexto hospitalario, ha considerado la satisfacción usuaria de la competencia de comunicación enfermera(o) -paciente, en sus dimensiones de proactividad, actitud profesional, comunicación verbal, comunicación no verbal y apoyo emocional. Lo cual difiere de otros que se han orientado fundamentalmente a medir variables estructurales, eficiencia técnica y oportunidad de la atención (15-18, 21, 22, 24, 43-45).

Existe un alto grado de satisfacción global de la comunicación del profesional de enfermería percibida por los pacientes de los centros de salud estudiados, sin embargo al realizar análisis desagregados de cada variable, el grado de satisfacción es menor, especialmente en lo referente a la comunicación no verbal $(17-19,23,26)$. Llama la atención que en un estudio realizado en Chile por la Superintendencia de Salud, medida la satisfacción usuaria en varias de sus dimensiones, los usuarios del sistema tanto público como privado, frente a la pregunta de cuál sería la "atención ideal" (sic), valoran en un nivel muy menor "que lo hagan sentir como persona", siendo superado ampliamente por el uso de tecnología de punta y la rapidez en la atención (11). En este mismo estudio el profesional de enfermería aparece entregando información derivada de la hospitaliza- 
ción y del proceso de alta muy por debajo de otros profesionales, en especial del médico, desaprovechando una instancia comunicativa-informativa que le permitiría al profesional establecer una relación satisfactoria para optimizar la recuperación y proyectar los cuidados domiciliarios y autocuidado de las personas, según sea el caso.

Dentro de los resultados encontrados, las mujeres perciben un mayor grado de insatisfacción de la comunicación enfermera-paciente, en relación a los hombres, esto podría deberse a que éstas son más participes en el proceso de salud-enfermedad, contribuyendo a que presenten más conocimiento de los aspectos relacionados con éste, generando de esta manera una mirada más crítica hacia los profesionales y la calidad percibida de la atención (11).

Los resultados muestran que existen diferencias de valoraciones acerca de la comunicación enfermera-paciente de acuerdo a la edad, coincidiendo con otros estudios referidos al contexto hospitalario, que señalan un alto porcentaje de satisfacción a medida que avanza la edad, particularmente en los adultos mayores, grupo donde se encuentran los más altos porcentajes de muy satisfecho (27). Este fenómeno se podría deber a que existen diferentes necesidades según la etapa de vida, privilegiando los jóvenes las habilidades técnicas e instrumentales para el cuidado que las comunicativas; en cambio los adultos mayores valoran mayormente las habilidades comunicativas del profesional de enfermería (14).

En este estudio, las personas que tienen una mayor estancia de 31 días y más perciben un mayor grado de satisfacción respecto a la comunicación enfermera-paciente, lo cual se contrapone con otras evidencias (11, 21,23), pudiéndose explicar que el factor de permanencia en el servicio permitiría que la comunicación enfermera-paciente se dé en mejores condiciones, por existir un mayor grado de conocimiento entre ambos actores.

En los servicios de medicina los pacien- tes presentan un mayor grado de insatisfacción, probablemente debido a que por una condición de salud más compleja demanda al profesional principalmente acciones técnicas e instrumentales para el cuidado, que comunicativas. Respecto a las competencias para el cuidado, Travelbee señala que una enfermera(o) es capaz de establecer la relación porque posee el conocimiento y las habilidades necesarias que se requieren para ayudar a las personas enfermas, siendo capaz de percibir, responder y apreciar la unicidad del ser humano enfermo (39), lo anterior demuestra que las competencias del profesional en cuanto a actitudes comunicativas son fundamentales para el establecimiento de una relación efectiva persona-persona y contribuir así a una adecuada satisfacción usuaria (34).

La cultura laboral predominante dentro de los servicios de salud es a mantener una relación distante con las personas atendidas, al otorgarle mayor importancia a las habilidades técnicas e instrumentales que a lo relacionado con el proceso comunicativo. El cuidado prestado se deshumaniza por las rutinas de trabajo, que impiden la visualización del otro como un ser integral, que tiene dudas y presenta angustia por su condición de salud y lo que éste requiere es calma, comprensión y apoyo $(2,6,7,28,37)$.

Los resultados expuestos para las áreas de comunicación verbal, actitud profesional, apoyo emocional y proactividad, muestran el alto grado de cumplimiento de las expectativas que presentan los usuarios en relación a las competencias comunicativas del profesional enfermero. A pesar de los resultados obtenidos a nivel global, se debe seguir desarrollando y potenciando las habilidades comunicativas desde la formación de los profesionales $(18,30,35,41)$. Uno de los hallazgos más relevantes en el estudio fue identificar falencias en la dimensión no verbal de la comunicación; según la literatura, la importancia radica en que esta área es la más influyente en la transmisión del mensaje, puesto que el cómo se comunica es lo que repercute 
de mayor forma en la respuesta por parte del usuario $(16,26,31,32,39-42)$.

La determinación del grado de satisfacción usuaria respecto a la competencia de comunicación del profesional de enfermería, en el contexto hospitalario, como indicador de calidad de la atención, ha permitido realizar una medición más precisa, utilizando de base algunos instrumentos cuantitativos y los principios de la teoría de Joyce Travelbee, que releva aspectos cualitativos en la calidad de los cuidados como es la comunicación enfermera-paciente. Este estudio pretende ser un aporte para la gestión de calidad intrahospitalaria y para mejorar las propias prácticas del profesional de enfermería, dándose un tiempo para establecer una real comunicación con las personas que permita identificar su rol del de otros profesionales en la gestión del cuidado.

Se torna de vital importancia considerar en la formación a la comunicación como una competencia profesional, no solamente definida en su carácter genérico, ya que ella es un medio para establecer cuidados acordes a las necesidades de las personas.

El buen trato humano y la visualización del otro como un ser único, autónomo, que tiene derecho a ser informado y tomar decisiones respecto a su salud, son áreas que deben ser respetadas y desarrolladas como características inherentes a la profesión de enfermería, atendiendo a la llamada a gestionar los cuidados, como lo plantea la Reforma en Salud en Chile, con un enfoque integral biopsicosocial y no solamente biologicista y tecnológico.

El instrumento diseñado, concebido para mediciones cuantitativas, no deja de lado la posibilidad de acercar a concepciones teóricas cualitativas que complementen la comprensión de la comunicación interpersonal enfermera(o)-persona atendida. Es importante considerar que los estudios abordan el tema de la calidad desde el punto de vista de estructura, de proceso, de observación sobre la práctica, revisión de registros, auditorias, de resultados, de eventos adversos, estudios que combinan procesos y resultados para demostrar los efectos del sistema, entre otros (14); sin embargo el aporte pretendido con el presente estudio es el diseño de un instrumento cuantitativo para medir un aspecto cualitativo como es la comunicación enfermera(o)-persona, en un contexto de cuidados médico-quirúrgicos de adultos, quedando abierta la posibilidad de utilizarlo en otros y así contribuir a darle una mayor potencia de validación, siguiendo los postulados de Nightingale quien ya había planteado en su época: "...existe necesidad inminente de conceptualizar a la enfermería acerca de su naturaleza y de la necesidad de desarrollar un cuerpo de conocimientos diferentes al de la medicina, por tanto la enfermería es cuidar y ayudar al paciente que sufre..." (8), ha pasado tanto tiempo y aún estamos discutiendo nuestro ser enfermero, proponiendo una de las estrategias para identificar las necesidades del otro a través de la comunicación.

Travelbee brinda las pautas para instalar lo que llama una revolución humanística que requiere que el profesional enfermera(o) integre al paciente en el proceso de recuperación para aliviar las aflicciones desencadenadas por las necesidades no atendidas. En este marco, el cuidado no solo se limita a realizar las prescripciones médicas, sino que principalmente a atender las necesidades individuales de cada persona como un camino hacia la comprensión del cuidar desde la perspectiva de la relación enfermera-paciente, donde la comunicación entre ambos es fundamental $(24,32,36,39,41)$.

\section{REFERENCIAS}

1. Gottlieb L, Rowat K. The McGill model of nursing: a practice-derived model. ANS Adv Nurs Sci. 1987; 9(4): 51-61.

2. Bouwman E, Sanson, I, Carvalho D. Relacionamento enfermeiro-paciente no 
pré-operatório: uma reflexão à luz da teoria de Joyce Travelbee. Cogitare Enferm. 2006; 11(1): 55-60.

3. Rivera L, Triana, Á. Percepción de comportamientos de cuidado humanizado de enfermería en la Clínica del Country. av. enferm. 2007; XXV (1): 56-68.

4. Canovaca A. Perfil Comunicacional del Médico de Atención Primaria en las consultas de demanda: Validación de un cuestionario [Tesis Doctorado]. [Córdoba (AR)]: Universidad de Córdoba; 2010. $130 \mathrm{p}$.

5. Ramírez C, Parra M. Percepción de los comportamientos del cuidado de enfermería en la unidad de cuidados intensivos. av.enferm. 2011; XXIX (1): 97-108.

6. Campos A. Importancia de las relaciones interpersonales en los cuidados de enfermería. Reporte presentado en el I Congreso Internacional de Enfermería [Internet]. Querétaro: Universidad del Valle de México; 2006 [citado 5 abril 2010]. Disponible en: http://www.uvmnet.edu/ investigacion/episteme/numero8y906/ enfoque/a_relaciones.asp

7. Aparicio G, Hernández A, Ostiguín R. Intersubjetividad: esencia humana del cuidado profesional de enfermería. Revista Enfermería Universitaria ENEO-UNAM [Internet] 2008 [citado 24 agosto 2013]; 5(1): 45-48. Disponible en: http://www. revistas.unam.mx/index.php/reu/article/ view/30275

8. Nightingale F. Notas sobre enfermería: qué es y qué no es. Barcelona: Elsevier Masson; 1990: $1^{a}$ impresión. Traducción al español de Sor Josefina Castro Vizoso. Hospital Universitario de Granada p. 141.

9. Travelbee J. Intervención en enfermería Psiquiátrica: el proceso de la relación persona a persona. 2a ed. OPS-OMS; 1982. pp. 46-94.

10. Rocha T, Faría S. La comunicación enfermera-cliente en el cuidado en las unidades de urgencia 24 h: una interpretación en Travelbee. Enferm. Glob [Internet].
2013 [citado 24 agosto 2013]; 12(30): 76-90. Disponible en: http://scielo.isciii.es/scielo.php?script=sci_arttext\&pi$\mathrm{d}=$ S1695-61412013000200005

11. Superintendencia de Salud de Chile. Estudio de usuarios del sistema de salud y prestadores respecto a la ley de derechos y deberes del paciente [Internet]. Chile: Superintendencia de Salud Chile; 2014 enero [citado 21 octubre 2014]. 102 p. Disponible en: http://www.supersalud.gob.cl/documentacion/569/articles-9820_recurso_1.pdf

12. Bastías G, Valdivia G. Reforma de salud en Chile; el plan AUGE o régimen de garantías explícitas en salud (GES). Su origen y evolución. Bol. Esc. Med. PUC [Internet]. 2007 [citado 26 marzo 2012]; 32(2): 51-58. Disponible en: http://escuela.med.puc.cl/publ/boletin/20072/ ReformaSalud.pdf

13. Superintendencia de Salud de Chile. Manual del estándar general de acreditación para prestadores institucionales de atención cerrada [Internet]. Santiago: Superintendencia de Salud de Chile; 2009 [citado 24 agosto 2013]. 49 p. Disponible en: http://www.supersalud.gob.cl/documentacion/569/articles-7132_recurso_1. pdf

14. Donabedian A. La calidad de la atención médica. Rev Calidad Asistencial. 2001; 16 (Supl 1): S29-S38.

15. Bustamante J., Órdenes F. Análisis de la percepción sobre satisfacción de los usuarios en el Hospital Regional de Valdivia [cybertesis]. (Chile): Universidad Austral de Chile; 2003.45 p. [citado 9 marzo 2013]; Disponible en: http://cybertesis. uach.cl/tesis/uach/2003/feb982a/doc/feb982a.pdf

16. Cabrera G, Bello L, Londoño J. Calidad percibida por usuarios de hospitales del programa de reestructuración de redes de servicios de salud de Colombia. Rev. Salud pública. 2008; 10(4): 593-604.

17. Barragán J, Moreno C. Calidad percibida 
por usuarios de enfermería en tres hospitales públicos. Enferm. Glob [Internet]. 2013 [citado 9 marzo 2013]; 12(29): 217-230. Disponible en: http://scielo.isciii.es/ scielo.php?script=sci_arttext\&pi$\mathrm{d}=$ S1695-61412013000100011

18. Gómez M, Feijoo M. Calidad de atención en salud percibida por los pacientes en un centro de salud urbano de A Coruña. Enferm Clin. 2012; 22(4): 182-190.

19. Jélvez A, Riquelme Y, Gómez N. Evaluación de la calidad de servicio en centro de salud familiar en Chile. Horizontes empresariales. 2010; 9(2): 51-65.

20. Departamento de Participación Social y Trato al Usuario (Subsecretaría de Redes Asistenciales). Medición nacional de satisfacción usuaria en la red pública de salud de Chile. Aplicación 2009 [Internet]. Santiago: Departamento de Participación Social y Trato al Usuario; 2009 diciembre [acceso 26 agosto 2013]. 102 p. Disponible en: http://www.ssmso.cl/tmparchivos/transparente/ParticipacionCiudadana/SatisfaccionUsuaria/MEDICIONNACSATISFAC.pdf

21. Tamargo T, Jiménez R, Gutiérrez Á, Mora I. Estadía hospitalaria ajustada para evaluar la eficiencia en un servicio de medicina interna. Rev cubana Med. 2009; 48(2): [16 p].

22. Bautista LM. Percepción de la calidad del cuidado de enfermería en la ESE Francisco de Paula Santander. Aquichan. 2008; 8(1): 74-84.

23. Massip C, Ortiz R, Llantá M, Peña M, Infante I. La evaluación de la satisfacción en salud: un reto a la calidad. Rev Cub Salud Pública. 2008; 34(4).

24. Riveros J, Berné C. Análisis de la opinión de usuarios sobre calidad percibida $y$ satisfacción con hospitales públicos: estudio de caso desde la perspectiva de la aplicación del marketing. Rev Med Chil. 2007; 135(7): 862-870.

25. Rulicki S, Cherny M. Comunicación no verbal: cómo la inteligencia emocional se expresa a través de los gestos. Buenos Aires: Ediciones Granica; 2007. 189 p.

26. Cepeda M, Piñero M. Calidad asistencial en la atención al paciente hospitalizado y su influencia en la satisfacción del usuario. Telos [Internet]. 2005 [acceso 26 agosto 2013 ]; 7(1):21-36. Disponible en: http://virtual.urbe.edu/artectexto/TEL/ TEL-015/TEL-015-002/texto.pdf

27. Santiñá MA, Martínez G, Quintó L, Trilla A, Asenjo M. Influencia de la edad del paciente en la percepción de la calidad asistencial. Rev Calid Asist. 2004; 19(4): 238-42.

28. Mejía M. Reflexiones sobre la relación interpersonal enfermera-paciente en el ámbito del cuidado clínico. Index Enferm. 2006; 15(54): 48-52.

29. Peplau $\mathrm{H}$. Interpersonal relations in nursing: a conceptual frame of reference for psychodynamic nursing. New York: Springer Publishing Company; 1991. 335 p.

30. Baker D, Hayes R, Fortier J. Interpreter use and satisfaction with interpersonal aspects of care for spanish-speaking patients. Med. Care [Internet]. 1998 [citado 24 agosto 2013]; 36(10): 1461-1470. Disponible en: http://www.ncbi.nlm.nih. gov/pubmed/9794340

31. Cibanal L. Técnicas de comunicación y relación de ayuda en ciencias de la salud. 2a ed. Barcelona: Elsevier; 2010. 304 p.

32. Watzlawick P, Beavin J, De Ávila J. Teoría de la comunicación humana: interacciones, patologías y paradojas. Buenos Aires: Editorial Tiempo Contemporáneo; 1971. $258 \mathrm{p}$.

33. Kéroauc S, Pepin J, Ducharme A, Major F. El pensamiento enfermero. Barcelona: Elsevier Masson; 2007. 167 p.

34. Falcó A. La nueva formación de profesionales: sobre la competencia profesional y la competencia del estudiante de enfermería. Educ. méd. 2004; 7(1): 42-45.

35. Bowen S. Language barriers in access to health care [Internet].Ottawa: Publica- 
tions Health Canada; 2001 Nov [citado 24 agosto 2013]. 120 p. Disponible en: http://www.hc-sc.gc.ca/hcs-sss/alt_formats/hpb-dgps/pdf/pubs/2001-lang-acces/2001-lang-acces-eng.pdf

36. González Y. La enfermera experta y las relaciones interpersonales. Aquichan. 2007; 7(2): 130-138.

37. Otero H. Hacia una comunicación efectiva y humanista en ámbitos de salud. Rev haban cienc méd [Internet]. 2008 Mar [acceso 24 agosto 2013]; 7(1). Disponible en: http://scielo.sld.cu/scielo.php?pi$\mathrm{d}=$ S1729-519X2008000100002\&scrip$\mathrm{t}=$ sci_arttext

38. Torres A, Sanhueza O. Desarrollo de la autoestima profesional en enfermería. Invest Educ Enferm. 2006; 24(2): 112119.

39. Raile M. Modelos y teorías en enfermería. $7^{\mathrm{a}}$ ed. Barcelona: Elsevier; 2011. 797 p.

40. Ortiz R, Muñoz S, Torres E. Satisfacción de los usuarios de 15 hospitales de Hidalgo, México. Rev. Esp. Salud Pública. 2004; 78(4): 527-537.

41. Montagu A. El tacto: la importancia de la piel en las relaciones humanas. Barcelona: Ediciones Paidós; 2004. 541 p.

42. Carvalho A, Arruda I, Costa I. Comunicación terapéutica en enfermería: herramienta esencial de la atención. Rev Bras Enferm. 2008; 61(3): 312-318.

43. Beltrán-Salazar O. La práctica de enfer- mería en cuidado intensivo. Aquichan. 2008; 8(1): 51-63.

44. Castillo L, Dougnac A, Vicente I, Muñoz V, Rojas V. Los predictores de satisfacción de pacientes en un centro hospitalario universitario. Rev Med Chil. 2007; 135(6): 696-701.

45. Simón P, Barrio I, Sánchez C, Tamayo M, Molina A, Suess A, et al. Satisfacción de los pacientes con el proceso de información, consentimiento y toma de decisiones durante la hospitalización. An Sist Sanit Navar. 2007; 30(2): 191-198.

46. Pons X. La comunicación entre el profesional de la salud y el paciente: aspectos conceptuales y guía de aplicación. Enferm. integr [Internet]. 2006 Mar [citado 9 marzo 2013]; (73): 27-34. Disponible en: http://www.enfervalencia.org/ei/73/ articulos-cientificos/ac_6.pdf

47. Rodríguez I, Hernández M. Lenguaje no verbal. La Coruña: Netbiblo; 2010. 160 p.

48. Watson J. Assessing and Measuring Caring in Nursing and Health Science. New York: Springer Publishing Company; 2002: 25-27, 50-52. 368 p.

49. López V, Sánchez J, Toronjo A, Pedregal M, Rojas M, Contreras A. Valoración del componente comunicativo/relacional de la práctica enfermera en Atención Primaria. El cuestionario GATHA-ENFERMERÍA. Index Enferm. 2008; 17(3): 173177. 